\title{
A 15.65-solar-mass black hole in an eclipsing binary in the nearby spiral galaxy M 33
}

\section{Citation}

Orosz, Jerome A., Jeffrey E. McClintock, Ramesh Narayan, Charles D. Bailyn, Joel D. Hartman, Lucas Macri, Jiefeng Liu, et al. 2007. "A 15.65-Solar-Mass Black Hole in an Eclipsing Binary in the Nearby Spiral Galaxy M 33." Nature 449 (7164): 872-75. https://doi.org/10.1038/nature06218.

\section{Permanent link}

http://nrs.harvard.edu/urn-3:HUL.InstRepos:41384888

\section{Terms of Use}

This article was downloaded from Harvard University's DASH repository, and is made available under the terms and conditions applicable to Other Posted Material, as set forth at http:// nrs.harvard.edu/urn-3:HUL.InstRepos:dash.current.terms-of-use\#LAA

\section{Share Your Story}

The Harvard community has made this article openly available.

Please share how this access benefits you. Submit a story.

Accessibility 


\section{A $15.65 M_{\odot}$ black hole in an eclipsing binary in the nearby spiral galaxy Messier 33}

Jerome A. Orosz ${ }^{1}$, Jeffrey E. McClintock ${ }^{2}$, Ramesh Narayan², Charles D. Bailyn ${ }^{3}$, Joel D. Hartman², Lucas Macri ${ }^{4}$, Jiefeng Liü ${ }^{2}$, Wolfgang Pietsch ${ }^{5}$, Ronald A. Remillard ${ }^{6}$, Avi Shporer ${ }^{7}$ \& Tsevi $\operatorname{Mazeh}^{7}$

${ }^{1}$ Department of Astronomy, San Diego State University, 5500 Campanile Drive, San Diego, CA 92182-1221, USA.

${ }^{2}$ Harvard-Smithsonian Center for Astrophysics, 60 Garden Street, Cambridge, MA 02138, USA.

${ }^{3}$ Department of Astronomy, Yale University, PO Box 208101, New Haven, CT 06520-8101, USA.

${ }^{4}$ National Optical Astronomy Observatory, 950 North Cherry Avenue, Tuscon, AZ 85719, USA.

${ }^{5}$ Max-Planck-Institut für extraterrestrische Physik, Giessenbachstraße, D-85741 Garching, Germany.

${ }^{6}$ MIT Kavli Institute for Astrophysics and Space Research, 77 Massachusetts Avenue, 37-287, Cambridge, MA 02139, USA.

${ }^{7}$ Wise Observatory, Tel Aviv University, Tel Aviv 69978, Israel.

Stellar-mass black holes are discovered in X-ray emitting binary systems, where their mass can be determined from the dynamics of their companion stars ${ }^{1-3}$. Models of stellar evolution have difficulty producing black holes in close binaries with masses $>10 M_{\odot}($ ref. 4), which

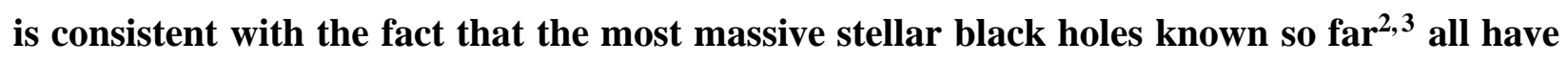
masses within $1 \sigma$ of $10 M_{\odot}$. Here we report a mass of $15.65 \pm 1.45 M_{\odot}$ for the black hole 
in the recently discovered system M33 X-7, which is located in the nearby galaxy Messier 33 (M33) and is the only known black hole that is in an eclipsing binary ${ }^{5}$. In order to produce such a massive black hole, the progenitor star must have retained much of its outer envelope until after helium fusion in the core was completed ${ }^{4}$. On the other hand, in order for the black hole to be in its present 3.45 day orbit about its $70.0 \pm 6.9 M_{\odot}$ companion, there must have been a "common envelope" phase of evolution in which a significant amount of mass was lost from the system ${ }^{6}$. We find the common envelope phase could not have occured in M33 X-7 unless the amount of mass lost from the progenitor during its evolution was an order of magnitude less than what is usually assumed in evolutionary models of massive stars $^{7-9}$.

Optical imaging and spectroscopic observations of M33 X-7 were obtained in service mode with the 8.2m Gemini North Telescope between 2006 August 18 and November 16. The mean optical spectrum is shown in Figure 1. The radial velocities derived from the 22 usable spectra show a nearly sinusoidal variation when phased on the orbital period of 3.453014 days determined from the X-ray eclipses ${ }^{5}$ (Fig. 2b).

Time series photometry was derived from the Gemini images in the Sloan $\mathrm{g}^{\prime}$ and $\mathrm{r}^{\prime}$ filters (Fig. 3). Additional photometric data were obtained in the $B, V$, and $I$ filters using the $3.5 \mathrm{~m}$ WIYN telescope during 2006 August 18-21 and 2006 September 15-16 (see Supplementary Information). The phased light curves show the characteristic ellipsoidal variations of a tidally distorted star (Fig. 3), which have been reported previously for this source ${ }^{10,11}$.

The temperature of the companion star was determined by comparing its averaged spectrum 
(Fig. 1) to a collection of synthetic spectra derived from the OSTAR2002 grid $^{12}$. As is usually the case, there is a strong correlation in the library of models between effective temperature, $T_{\text {eff }}$, and surface gravity, $\log g$, and thus various combinations of these parameters result in very similar spectra. Fortunately, the dynamical information strongly constrains the allowed value of $\log g$ to be between 3.65 and 3.75 at the $3 \sigma$ level. A good match to the observed spectrum is provided by the model with $T_{\text {eff }}=35000 \mathrm{~K}, \log g=3.75$, a metalicity of $10 \%$ of the solar value (representative of star clusters in M 33 at this galactocentric distance ${ }^{13}$ ), and our measured value of the projected rotational velocity of $V_{\mathrm{rot}} \sin i=250 \pm 7 \mathrm{~km} \mathrm{~s}^{-1}$ (where $i$ is the orbital inclination angle). The formal error on the temperature is $\pm 200 \mathrm{~K}$ for $\log g=3.75$. However, given the possibility of a gravity slightly different than $\log g=3.75$ (the grid spacing of the models is 0.25 dex) and the correlation between $T_{\text {eff }}$ and $\log g$, we adopt a temperature in the range $34000 \leq T_{\text {eff }} \leq 36000 \mathrm{~K}$, which corresponds to a spectral type of O7III to O8III ${ }^{14}$.

To compute the radius of the O-star, we adopt a distance modulus of $24.62 \pm 0.05 \mathrm{mag}$ $(d=840 \pm 20 \mathrm{kpc}$ ) to M33 (see the Supplementary Information for details). Using $V=18.9 \pm 0.05$ $A_{V}=0.53 \pm 0.06 \mathrm{mag}$ (ref. 10), and the bolometric corrections derived from the OSTAR2002 grid, we find a radius of $R_{2}=19.6 \pm 0.9 R_{\odot}$ and a luminosity of $\log \left(L / L_{\odot}\right)=5.72 \pm 0.07$.

The duration of the X-ray eclipse $\Theta$ (measured in degrees of orbital phase) strongly constrains the available parameter space. Because the size of the compact X-ray source ( $\lesssim 1000 \mathrm{~km})$ is vastly smaller than the secondary star, one might expect the X-ray eclipse profile to be a "square well" with a flat bottom and very abrupt periods of ingress and egress. However, in M33 X-7 
and other X-ray binaries with massive companions, the observed eclipse profile deviates from this idealised picture. The transitions into and out of eclipse are more gradual because of absorption of the X-rays in the stellar wind that thickens near the O-star. The non-zero X-ray intensity in full eclipse results from X-rays that are scattered by this wind around the O-star into our line-of-sight. The erratic X-ray variability prior to eclipse (Fig. 2a) is presumably caused by absorption in the gas that is streaming from the $\mathrm{O}$-star to the black hole. The period of egress is free of such effects, and we focus our attention there. We identify the eclipse width of $\Theta=53 \pm 2.2^{\circ}$ (ref. 5) as the onset of the steep egress feature (solid line in Fig. 2a). In the Supplementary Information, we show that the eclipse width of $\Theta=53^{\circ}$ is consistent with absorption in the stellar wind, whereas the true eclipse by the stellar photosphere corresponds to $\Theta=46 \pm 1^{\circ}$ (dashed-dotted line in Fig. 2a).

We used a light curve synthesis code ${ }^{15}$ to find the optimal model of the binary system. Fig. 3 shows the synthetic light curves for the best-fitting model, which is schematically illustrated in Fig. 4. The best-fitting model parameters and derived astrophysical parameters are summarised in Table 2. The mass of the compact object is $M=15.65 \pm 1.45 M_{\odot}$, and the mass of the O-star is $M_{2}=70.0 \pm 6.9 M_{\odot}$, which puts it among the most massive stars whose masses are well-determined ${ }^{16}$. The effective radii of the Roche lobes are $21.8 R_{\odot}$ and $10.8 R_{\odot}$ for the O-star and black hole, respectively. From evolutionary models of single stars ${ }^{7-9}$ the age of the O-star is estimated to be between about 2 and 3 million years. We also note in passing that the O-star is roughly a factor of three less luminous than expected from the evolutionary models.

With $M=15.65 \pm 1.45 M_{\odot}, \mathrm{M} 33 \mathrm{X}-7$ is the most massive stellar black hole known (see 
Table 1). The mass of V404 Cyg is $12 \pm 2 M_{\odot}$ and the masses of the 18 other black holes, save one, are $\lesssim 10 M_{\odot}$, or they are quite imprecise. The one contender is GRS 1915+105 with a mass of $14.0 \pm 4.4 M_{\odot}($ refs. 17,18$)$. However, the $30 \%$ precision of the measurement is poor. Furthermore, there are reasons for questioning the reliability of this impressive and pioneering result on a difficult system. For example, the spectroscopic orbital period ${ }^{17}$ is $9 \%$ longer than the recently-determined and precise photometric period ${ }^{19}$. Furthermore, because of the large X-ray luminosity, the latetype secondary star contributes "only a few per cent of the K-band brightness ${ }^{17}$;" hence, the radial velocity curve may be significantly distorted ${ }^{20}$. By comparison, the mass estimate and eclipse ephemeris for M33 X-7 are exceptionally precise, and X-ray heating is a minor effect.

M33 X-7 is a key system in the study of high mass stars, high mass X-ray binaries, and high mass black holes. $\mathrm{A} \approx 16 M_{\odot}$ black hole paired with $\mathrm{a} \approx 70 M_{\odot}$ secondary with a separation of only $\approx 42 R_{\odot}$ is very difficult to explain using stellar evolutionary models. Since the radius of the black hole progenitor would have been much larger than the current orbital separation ${ }^{7-9}$, the two stars must have been brought closer together via some kind of "common envelope" phase which results in a significant amount of mass lost from the progenitor, and very little mass gained by the secondary ${ }^{6}$. On the other hand, in order for the core mass to remain large enough to produce a $\approx 16 M_{\odot}$ black hole, the outer envelope of the progenitor needs to be intact until core He burning is completed $^{4}$. Hence we require that the common envelope phase begins only after core He burning in the progenitor is complete (case $\mathrm{C}$ mass transfer ${ }^{6}$ ). There are two requirements for a common envelope phase to start during case $\mathrm{C}$ mass transfer. First, the mass donor needs to be at least 1.2 times more massive than the secondary at the start of mass transfer ${ }^{21}$. Second, the radius of 
the mass donor at the end of core He burning needs to be larger than its radius at the end of core $\mathrm{H}$ burning. If the second condition is not met, the $\mathrm{CE}$ phase begins before core He burning is complete, and the stripped core loses much of its mass via strong winds in its subsequent evolution and thus cannot make a massive black hole ${ }^{4}$.

Assuming no large change in the present-day mass loss rate of $2.6 \times 10^{-6} M_{\odot} \mathrm{yr}^{-1}$ (see Supplementary Information), the secondary star has lost between about $5.2 M_{\odot}$ and $7.8 M_{\odot}$, thereby putting its initial mass near $\approx 80 M_{\odot}$. For a common envelope phase to occur, the progenitor star should have been more massive than $\approx 80 \times 1.2=96 M_{\odot}$, which is problematic. According to evolutionary models ${ }^{7-9}$, massive stars lose much of their initial mass via winds, and the mass loss rate generally increases with increasing initial mass. For example, even in the extreme case of an initial mass of $120 M_{\odot}$ and a metallicity of $20 \%$ solar, the mass after $\mathrm{H}$ burning is $\approx 52.9 M_{\odot}$ and after He burning is $\approx 17.2 M_{\odot}$ (ref. 9). Furthermore, owing to the large amount of mass loss, the radius of the star after core He burning is smaller than the radius after core $\mathrm{H}$ burning. For these reasons, a common envelope phase during case $\mathrm{C}$ mass transfer seems very unlikely. It would appear that the progenitor star of M33 X-7 lost roughly an order of magnitude less mass before the common envelope phase ensued than is predicted by the evolutionary models. Finally we note that there is an additional complication: even if a common envelope is formed, the most likely outcome would be a merger since the envelopes of massive stars are tightly bound ${ }^{21}$. However, the most massive star considered in ref. 21 was $50 M_{\odot}$, so the detailed computations should be extended to the higher masses relevant for M33 X-7. 
The determination of an accurate mass for M33 X-7 - located at a distance of more than 16 times that of any other confirmed stellar black hole - marks a major advance in our capability to study black holes in Local Group galaxies beyond the Milky Way.

1. Remillard, R. A. \& McClintock, J. E. X-Ray properties of black-hole binaries. Annu. Rev. Astron. Astrophys. 44, 49-92 (2006).

2. Charles, P. A. \& Coe, M. J. in Compact Stellar X-ray Sources (eds. W. H. G. Lewin \& M. van der Klis) 215-265 (Cambridge University Press, Cambridge, 2006).

3. Orosz, J. A. in A Massive Star Odyssey: From Main Sequence to Supernova (eds. van der Hucht, K. A., Herrero, A. \& Esteban, C.) Proc. IAU Symp. 212 365-371 (ASP, San Francisco, 2003).

4. Brown, G. E. Heger, A. Langer, N. Lee, C. -H. Wellstein, S., \& Bethe, H. A. Formation of high mass X-ray black hole binaries. New Astron. 6, 457-470 (2001).

5. Pietsch, W. et al. M33 X-7: ChASeM 33 reveals the first eclipsing black hole X-Ray binary. Astrophys. J. 646, 420-428 (2006).

6. Tauris, T. M. \& van den Heuvel, E. P. J. in Compact Stellar X-ray Sources (eds. W. H. G. Lewin \& M. van der Klis) 623-665 (Cambridge University Press, Cambridge, 2006).

7. Schaller, G., Schaerer, D., Meynet, G., \& Maeder, A. New grids of stellar models from 0.8 to $120 M_{\odot}$ at $Z=0.020$ and $Z=0.001$. Astron. Astrophys. Suppl. 96, 269-331 (1992). 
8. Meynet, G., Maeder, A., Schaller, G., Schaerer, D., \& Charbonnel, C. Grids of massive stars with high mass loss rates. V. From 12 to $120 M_{\odot}$ at $\mathrm{Z}=0.001,0.004,0.008,0.020$ and 0.040 . Astron. Astrophys. Suppl. 103, 97-105 (1994).

9. Vázquez, G. A., Leitherer, C., Schaerer, D., Meynet, G. \& Maeder, A. Models for massive stellar populations with rotation. Astrophys. J. 663, 995-1020 (2007).

10. Pietsch, W., Mochejska, B. J., Misanovic, Z., Haberl, F., Ehle, M. \& Trinchieri, G. The eclipsing massive X-ray binary M33 X-7: New X-ray observations and optical identification. Astron. Astrophys. 413, 879-887 (2004).

11. Shporer, A., Hartman, J., Mazeh, T., \& Pietsch, W. Photometric analysis of the optical counterpart of the black hole HMXB M33 X-7. Astron. Astrophys. 462, 1091-1095 (2007).

12. Lanz, T. \& Hubeny, I. A Grid of non-LTE line-blanketed model atmospheres of O-type stars. Astrophys. J. Suppl. 146, 417-441 (2003).

13. Ma, J. et al. Spectral energy distributions, ages, and metallicities of star clusters in M 33. Astron. J. 122, 1796-1806 (2001).

14. Heap, S. R., Lanz, T., \& Hubeny, I. Fundamental properties of O-type stars. Astrophys. J. 638, 409-432 (2006).

15. Orosz, J. A. \& Hauschildt. P. H. The use of the NextGen model atmospheres for cool giants in a light curve synthesis code. Astron. Astrophys. 364, 265-281 (2000). 
16. Geis, D. R. in A Massive Star Odyssey: From Main Sequence to Supernova (eds. van der Hucht, K. A., Herrero, A. \& Esteban, C.) Proc. IAU Symp. 212 91-100 (ASP, San Francisco, 2003).

17. Greiner, J., Cuby, J. G., \& McCaughrean 2001. An unusually massive stellar black hole in the galaxy. Nature 414, 522-525 (2001).

18. Harlaftis, E. T. \& Greiner, J. The rotational broadening and the mass of the donor star of GRS 1915+105. Astron. Astrophys. 414, L13-L16 (2004).

19. Neil, E. T., Bailyn, C. D., \& Cobb, B. E. Infrared monitoring of the microquasar GRS 1915+105: detection of orbital and superhump signatures, Astrophys. J. 657, 409-414 (2007).

20. Reynolds, A. P., Quaintrell, H., Still, M. D., Roche, P., Chakrabarty, D. \& Levine, S. E. A new mass estimate for Hercules X-1. Mon. Not. R. Astron. Soc. 288, 43-52 (1997).

21. Podsiadlowski, Ph., Rappaport, S., \& Han, Z. On the formation and evolution of black hole binaries. Mon. Not. R. Astron. Soc. 341, 385-404 (2003).

22. Lucy, L. B. \& Walsh, J. R. Iterative techniques for the decomposition of long-slit spectra. Astron. J. 125, 2266-2275 (2003).

23. Humphreys, R. M. \& Sandage, A. On the stellar content and structure of the spiral galaxy M 33. Astrophys. J. Suppl. 44, 319-381 (1980).

24. Alard, C. Image subtraction using a space-varying kernel. Astron. Astrophys. Suppl. 144, 363$370(2000)$. 
25. Stetson, P. B. DAOPHOT - A computer program for crowded-field stellar photometry. Pub. Astron. Soc. Pac. 99, 191-222 (1987).

Acknowledgements We thank J. Walsh for his help with the SPECRES software, I. Hubeny for the use of his model atmosphere codes, and T. Matheson for support with the Gemini Observations. CDB acknowledges support from the US National Science Foundation.

Competing Interests The authors declare that they have no competing financial interests.

Correspondence Correspondence and requests for materials should be addressed to J.A.O.

(email: orosz@sciences.sdsu.edu). 


\begin{tabular}{cccc}
\hline X-ray source & Optical/IR counterpart & Black hole mass $\left(M_{\odot}\right)$ & Secondary star mass $\left(M_{\odot}\right)$ \\
\hline \hline GRS 1915+105 & V1487 Aql & $14.0 \pm 4.4$ & $0.81 \pm 0.53$ \\
GS 2023+338 & V404 Cyg & $12 \pm 2$ & 0.6 \\
A0620-00 & V616 Mon & $10 \pm 5$ & 0.6 \\
GS 2000+25 & QZ Vul & $10 \pm 4$ & 0.5 \\
XTE J1550-564 & V381 Nor & $9.6 \pm 1.2$ & $\ldots$ \\
4U 1543-47 & IL Lup & $9.4 \pm 1.1$ & 2.5 \\
Cyg X-1 & HDE 226868 & $>4.8$ & $>11.7$ \\
LMC X-1 & $\ldots$ & $8-20$ & $\ldots$ \\
\hline
\end{tabular}

Table 1: Recent dynamical measurements of massive stellar black holes. Masses for GRS $1915+105$ are from ref. 18, and all others are taken from the compilation in ref. 2 and citations therein. The uncertainties correspond to one standard deviation. There are three key "observables" that can be used to determine the mass $M$ of the compact object in an X-ray binary. (1) The radial velocity semiamplitude of the secondary star $K_{2}$, along with the orbital period $P$ and eccentricity $e$, determines the mass function: $f(M)=$ $P K_{2}^{3}\left(1-e^{2}\right)^{3 / 2} /(2 \pi G)=M^{3} \sin ^{3} i /\left(M+M_{2}\right)^{2}$, where $M_{2}$ is the mass of the secondary star, $i$ is the orbital inclination angle and $G$ is the gravitational constant. In order to solve for $M$, we must determine $M_{2}$ (or $M_{2} / M$ ) and $i$, for which we use (2) the rotational velocity of the secondary star $V_{\text {rot }} \sin i$, and (3) the amplitude of the ellipsoidal light curve. The two preceding observables depend on $i, M_{2} / M$ and the Roche-lobe filling $f_{2}$, which is the radial fraction of the secondary's Roche equipotential lobe along the line of centers that is occupied by the star.

\begin{tabular}{rrrr}
\hline parameter & value & parameter & value \\
\hline \hline$\Theta(\mathrm{deg})$ & $46 \pm 1$ & $M_{2}\left(M_{\odot}\right)$ & $70.0 \pm 6.9$ \\
$T_{\text {eff }}(\mathrm{K})$ & $34000-36000$ & $r_{d}$ & $0.45 \pm 0.03$ \\
$V_{\text {rot }} \sin i\left(\mathrm{~km} \mathrm{~s}^{-1}\right)$ & $250 \pm 7$ & $e$ & $0.0185 \pm 0.0077$ \\
$R_{2}\left(R_{\odot}\right)$ & $19.6 \pm 0.9$ & $\omega(\mathrm{deg})$ & $140 \pm 27$ \\
$\log L_{2}\left(L_{\odot}\right)$ & $5.72 \pm 0.07$ & $\Omega$ & $0.903 \pm 0.037$ \\
$\Delta \phi$ & $0.0045 \pm 0.0014$ & $f_{2}$ & $0.777 \pm 0.017$ \\
$i(\mathrm{deg})$ & $74.6 \pm 1.0$ & $a\left(R_{\odot}\right)$ & $42.4 \pm 1.5$ \\
$K_{2}\left(\mathrm{~km} \mathrm{~s}^{-1}\right)$ & $108.9 \pm 5.7$ & $M\left(M_{\odot}\right)$ & $15.65 \pm 1.45$ \\
\hline
\end{tabular}

Table 2: Selected parameters for M33 X-7. The uncertainties correspond to one standard deviation. For the determination of $\Theta$, see the Supplementary Information. The measurements of $T_{\text {eff }}$ and $V_{\text {rot }} \sin i$ were derived directly from the spectra. $R_{2}$ and $\log L_{2}$ were derived from the temperature, apparent magnitude, extinction, and the distance. The next nine parameters were determined by fitting the radial velocity curve and light curves simultaneously using the ELC code ${ }^{15}$. The final two parameters are fixed by those given above. 

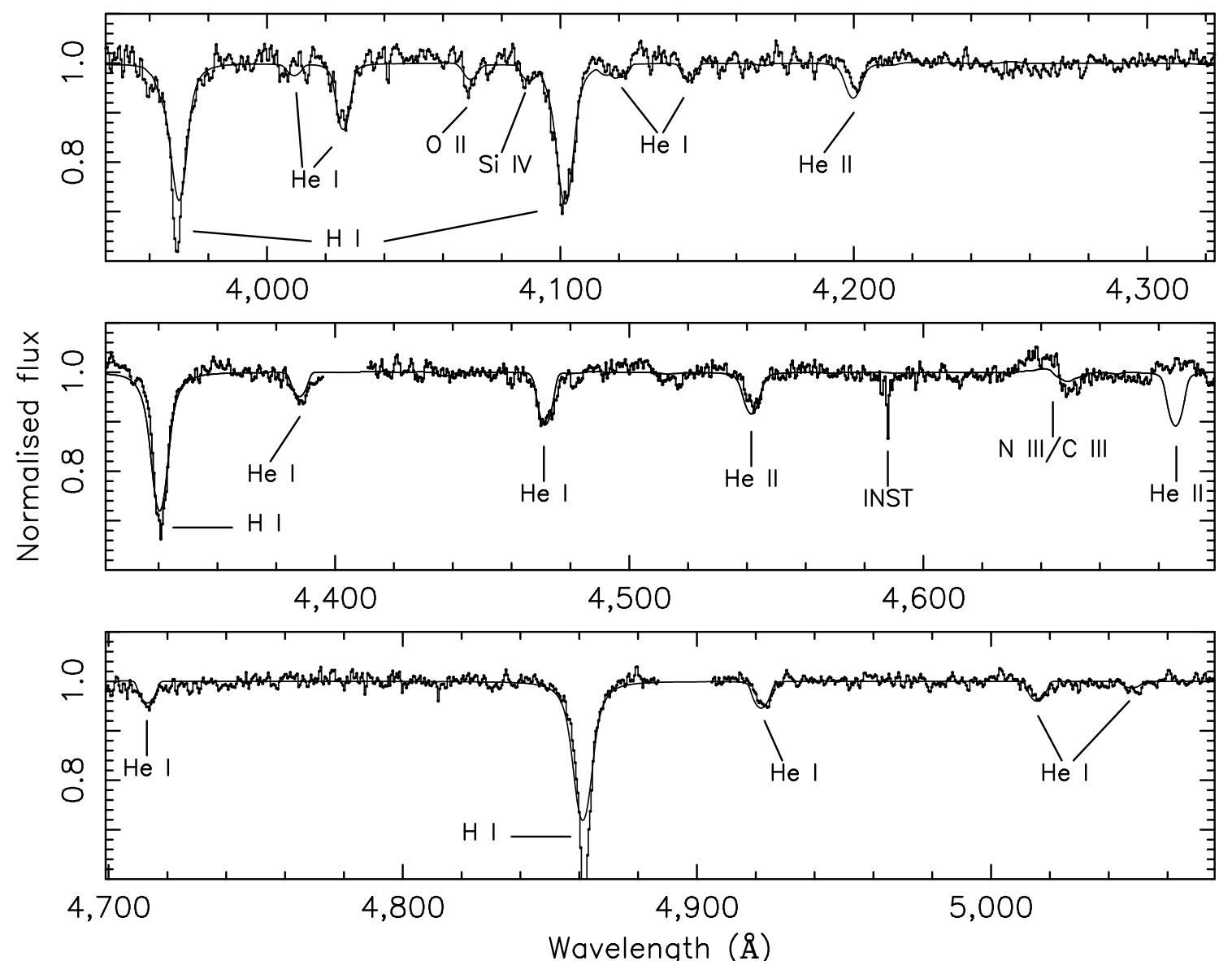

Figure 1 Mean optical spectrum of M33 X-7. The spectrum shown here (connected dots), which was extracted with the SPECRES package in IRAF ${ }^{22}$, is the sum of the 22 individual spectra that have been velocity-shifted to the rest frame of the secondary star. The solid line is the model spectrum described in the text. The data were obtained using the GMOS instrument on the $8.2 \mathrm{~m}$ Gemini-North Telescope with the B1200 grating $\left(\lambda_{c}=4650 \AA\right)$ and a 0.5 arcsecond slit rotated to a position angle of 215.6 degrees, which was defined by M33 X-7 and a nearby pair of stars 0.9 arcseconds to the southwest (see Supplementary Information). Twenty-four 40-minute spectra were acquired in service mode between 2006 August 18 and November 16 in good seeing of always $<0.8$ arcseconds. The two observations obtained on 2006 September 17 are suspect and will not be considered here. The initial bias subtraction, flat-fielding, and wavelength calibrations were performed using the GMOS package in IRAF. In the two-dimensional spectra, the overlap of the profiles of M33 X-7 and the nearby pair of stars was modest. The optimal extraction of one-dimensional spectra was done two ways. (1) Routines in the GMOS package were used with the spectral extraction aperture adjusted so that light from the nearby pair of stars was not included, which resulted in at most about a $20 \%$ loss of light from M33 X-7 (see Supplementary Information). The final extracted spectra had signal- 
to-noise ratios of 20 or more per $0.47 \AA$ pixel near $\mathrm{H} \beta$. Numerous nebular emission lines from the surrounding $\mathrm{HIl}$ region ${ }^{23}$ are seen in these spectra, including the Balmer lines $\mathrm{H} \beta$ through $\mathrm{H} \epsilon$, [O III] near 4363, 4969 and $5007 \AA$, and weak He I lines near 4026, 4471, 4921 and $5015 \AA$. The He II line near $4686 \AA$ and the N III lines near $4640 \AA$ are also in emission. The quality of the wavelength stability was checked by measuring the radial velocity of the brightest nebular line, [O III] 5007A. Its average heliocentric velocity in the 22 spectra is $-131.2 \pm 1.5 \mathrm{~km} \mathrm{~s}^{-1}$ (std. dev.); for comparison, the velocity of M33 in the NASA Extragalactic Database is $-179 \pm 3 \mathrm{~km} \mathrm{~s}^{-1}$. (2) Routines in the SPECRES package were used to deblend the spatial profiles of M33 X-7 and the nearby pair of stars and to remove the nebular lines before optimally extracting one-dimensional spectra. However, the resulting spectra have lower signal-to-noise ratios than the spectra extracted with the GMOS routines (see Supplementary Information). 

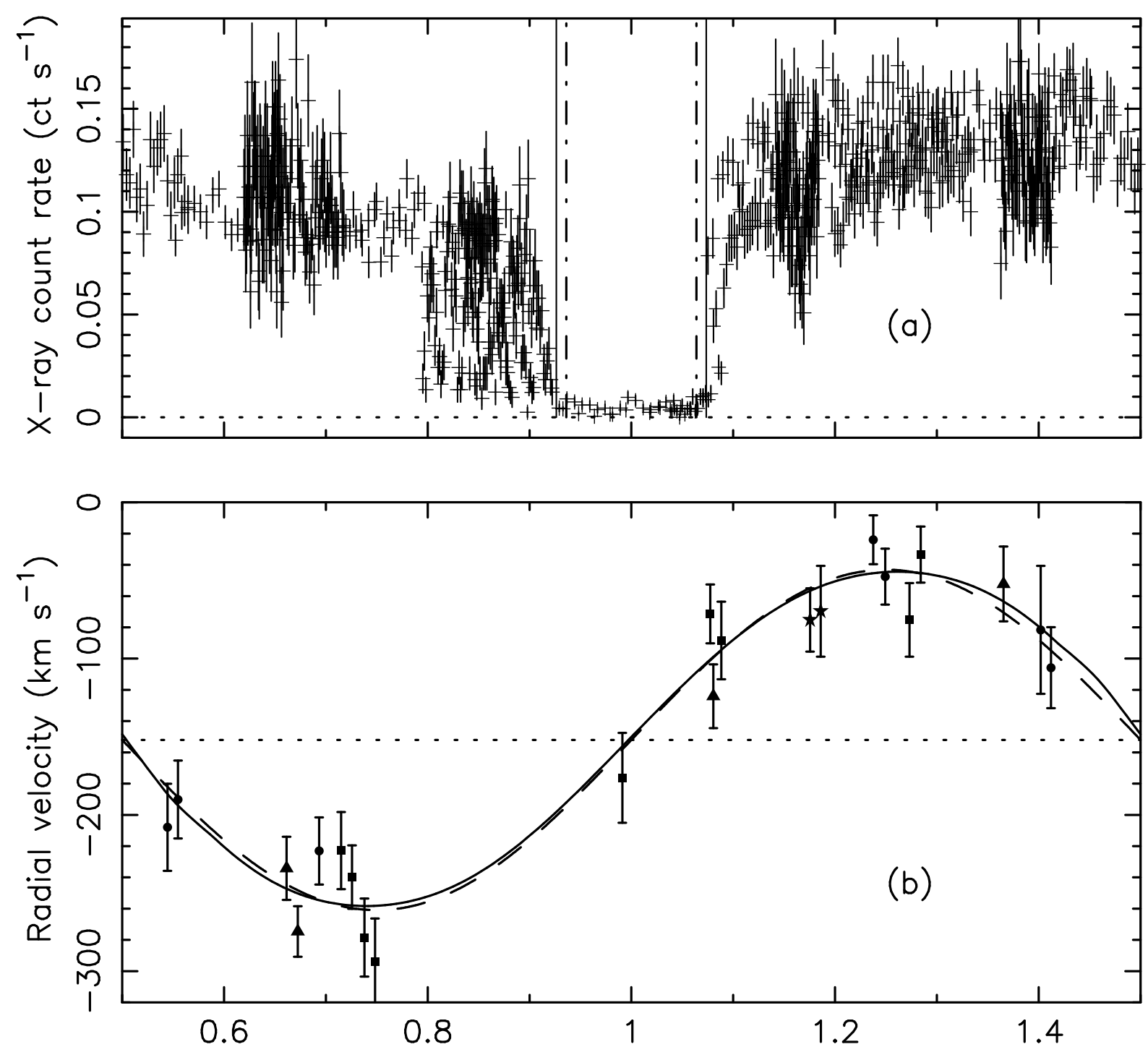

Orbital phase

Figure 2 Phased X-ray light curve and radial velocity curve for M33 X-7. (a) The Chandra ACIS light curve in the $0.5-5 \mathrm{keV}$ energy band. The error bars $1 \sigma$ (s.d.) statistical. Complete orbital phase coverage is achieved here using all 17 available ACIS observations, including five observations (ObsIDs 1730, 6376, 6385, 6387 and 7344) not present in Figure 1 of ref. 5. The count rates are corrected for vignetting and for the difference between the responses of the ACIS-S and ACIS-I detectors for the single ACIS-I observation (ObsID 6378). The solid vertical lines denote an X-ray eclipse duration of $\Theta=53^{\circ}$ (ref. $5)$, which incudes the effects of an extended wind from the O-star. The dash-dotted vertical lines denote an eclipse duration of $\Theta=46^{\circ}$, which corresponds solely to an eclipse by the photosphere of the O-star. (b) The radial velocity curve derived from the Gemini spectra (extracted using the GMOS IRAF package) with the best-fitting model shown 
as a solid line. The error bars $1 \sigma$ (s.d.) statistical. The dashed line is the best-fitting sinusoid. The radial velocities were derived by cross-correlating the spectra against a synthetic spectrum (Fig. 1) over the wavelength ranges 4150-4300 and 4521-4578 $\AA$. These bands include two He II lines, $4200 \AA$ and $4541 \AA$, which are uncontaminated by nebular lines. Radial velocities obtained in 2006 August are denoted by circles, 2006 September by squares, 2006 October by stars, and 2006 November by triangles. Using the orbital period of 3.453014 days determined from the X-ray eclipses ${ }^{5}$, a sine fit to the 22 velocities yields $K_{2}=108.9 \pm 6.4 \mathrm{~km} \mathrm{~s}^{-1}$, systemic velocity $\gamma=-152 \pm 5 \mathrm{~km} \mathrm{~s}^{-1}$, and $T_{0}=$ HJD $2,453,967.157 \pm 0.048$. Here, $T_{0}$ is the predicted time of mid-X-ray eclipse, which is in full agreement with that of ref. 5 - they differ by $95.001 \pm 0.014$ orbital cycles. The value of the mass function, which is the absolute minimum mass of the compact object, is $f(M)=0.46 \pm 0.08 M_{\odot}$. 


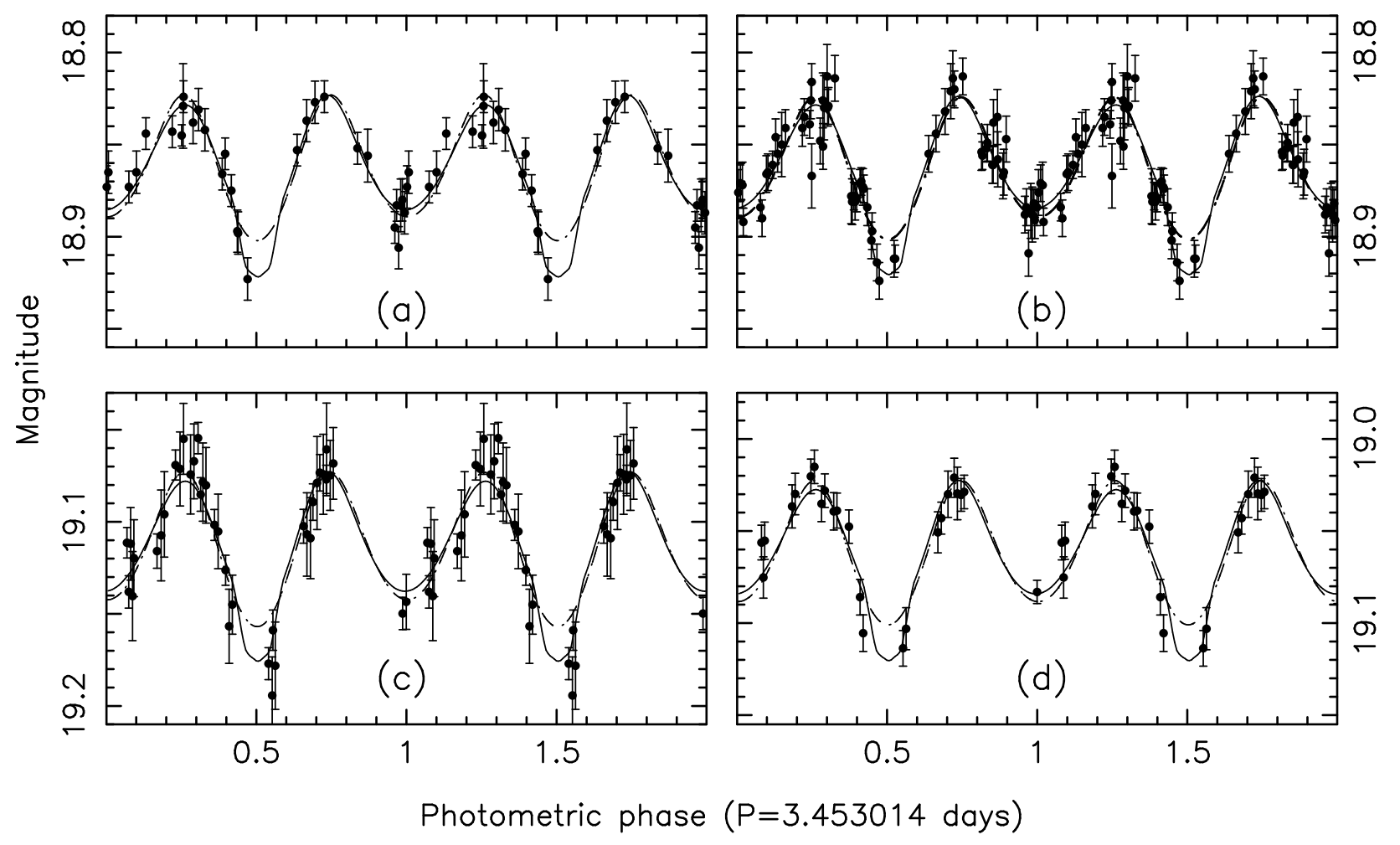

Figure 3 Optical light curves. (a) The $B$ light curve from ref. 10. (b) The $V$ light curve from ref. 10. (c) Gemini $g^{\prime}$ light curve. (d) Gemini $r^{\prime}$ light curve. The photometric time series were derived using the ISIS image subtraction software ${ }^{24}$ together with DAOPHOT ${ }^{25}$, which was used to determine the reference flux. The error bars are $1 \sigma$ (s.d.) statistical. The ELC code ${ }^{15}$ was used to find the optimal binary model. These light curves and the radial velocities shown in Fig. $2 \mathrm{~b}$ were used as input data. In addition, we have three other constraints: the radius of the O-star of $R_{2}=19.6 \pm 0.9 R_{\odot}$, the projected rotational velocity of the O-star of $V_{\text {rot }} \sin i=250 \pm 7 \mathrm{~km} \mathrm{~s}^{-1}$, and the width of the X-ray eclipse of $\Theta=46 \pm 1^{\circ}$ (see Supplementary Information). We note that the eclipse duration and known radius are strong constraints that are unavailable for Galactic black hole binaries. In deriving the models, we initially fitted for six parameters: $i, K_{2}, M_{2}$ (see the Table 1 caption for definitions), $R_{2}, T_{\text {eff }}$, and a phase shift $\Delta \phi$, which is used to account for small uncertainties in the ephemeris. The first three of these parameters, along with the established orbital period $P$, determine the scale of the binary, including the dimensions of the Roche equipotential lobes. The value of $R_{2}$ then determines the Roche-lobe filling factor $f_{2}$. With the geometry of the star fully specified, $T_{\text {eff }}$ and the gravity darkening law $\left(T \propto g^{1 / 4}\right)$ determines the distribution of temperatures over the surface the of the star. No parameterised limb darkening is required because we computed the specific intensities from the OSTAR2002 grid. Likewise, X-ray heating has been accounted for, and is anyway a minor correction $(\Delta T \leq 100 \mathrm{~K})$ because of the star's extreme luminosity. After several initial trial runs, we found that the fits were improved by (1) adding a faint accretion disk 
around the compact object with a fractional radius $r_{d}$, (2) allowing the orbit to be slightly non-circular (adds eccentricity $e$ and argument of periastron $\omega$ as free parameters), and (3) allowing the O-star to rotate slightly non-synchronously with the orbit, which is parameterised by $\Omega=P_{\text {orb }} / P_{\text {rot }}$ (we assume the star's rotation axis is perpendicular to the orbital plane). The solid lines show the best-fitting model, and the dash-dotted lines show the best-fitting models with a circular orbit and no accretion disk. The genetic optimiser code was run five times with different initial random parameter sets and the grid search optimiser was run many hundreds of times to refine the solution and define confidence limits on the fitted and derived parameters (see Supplementary Information). 


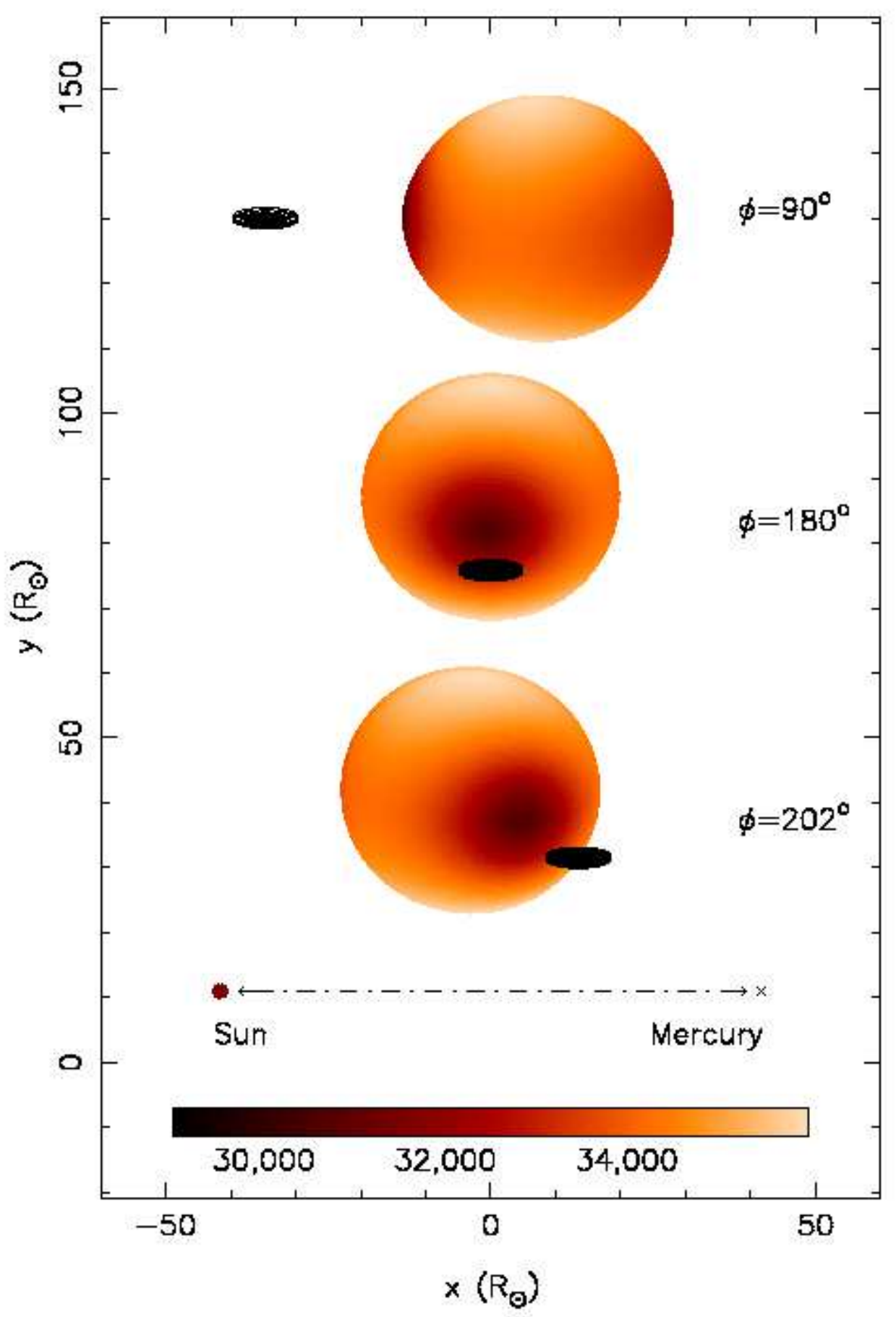

Figure 4 Schematic diagram of M33 X-7. The companion star and the accretion disk surrounding the black hole are shown to scale as seen projected onto the plane of the sky at three orbital phases. The colours on the star represent temperatures (not intensities), with cooler temperatures shown by darker colours as denoted on the bar. The distance between the Sun and Mercury is indicated and the figure is scaled in solar radii. 